\title{
Granzyme K inhibits replication of influenza virus through cleaving the nuclear transport complex importin $\alpha 1 / \beta$ dimer of infected host cells
}

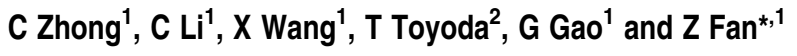

The influenza A virus is a causative agent of influenza, which infects human cells and uses host factors to accomplish viral genome replication as part of its life cycle. The nucleoprotein (NP) and PB2 of the influenza virus associate with importin $\alpha 1$ to gain access to the host nucleus through a ternary import complex. Killer cell-mediated cytotoxicity is the primary mechanism of eliminating the influenza virus. Here, we showed that lymphokine-activated killer cells participated in the elimination of the influenza virus. Granzyme (Gzm) K inhibition elevated viral replication in vitro and aggravated viral infection in vivo. We identified that importin $\alpha 1$ and its transport partner protein importin $\beta$ are physiological substrates of GzmK. Proteolysis of these two substrates wrecked their association to generate the importin $\alpha 1 / \beta$ dimer and disrupted transportation of viral NP to the nucleus, leading to inhibition of influenza virus replication.

Cell Death and Differentiation (2012) 19, 882-890; doi:10.1038/cdd.2011.178; published online 2 December 2011

Influenza A virus is a causative agent of influenza, a disease that results in devastating pandemics and global panic. The influenza $A$ virus is a negative-strand RNA virus containing eight segments of RNA as its genome. Transcription and replication of the viral genome are fulfilled in a host nucleus through RNAdependent RNA polymerase, which has three subunits, namely PB1, PB2, and PA. The nucleoprotein (NP) associates with viral RNAs (vRNA) to form a viral ribonucleoprotein (vRNP) complex to assist the polymerase in replicating the viral genome. It has been reported that the different subunits of RNA polymerase bind an importin $\alpha / \beta$ dimer or an importin $\beta$ homolog receptor of host cellular factors to gain access to the nucleus of the infected host cell for viral replication. ${ }^{1}$ Moreover, NP can also bind to importin $\alpha 1$ to enter the nucleus. ${ }^{2}$

Killer lymphocytes eliminate both viruses and cancer cells through granule-mediated cytotoxicity. ${ }^{3,4}$ These lymphocytes undergo exocytosis, releasing cytotoxic granules that release cytotoxic elements such as the pore-forming protein (PFP) perforin and granzymes (Gzms), which are taken up into the target cell by endocytosis. PFP actually helps Gzms enter the target cell, where they trigger apoptosis. ${ }^{5}$ Topham et al. ${ }^{6}$ reported that killer cell-mediated cytotoxicity is a primary mechanism of eliminating the influenza virus. More recently, Regner et al. ${ }^{7}$ reported that $g z m A \times B$ double deficiency $\left(\mathrm{gzmAB}^{-1-}\right)$ does not cause any impairment of infected target cell clearance. This suggests that other Gzms may participate in pathogenic elimination. Jenkins et al. ${ }^{8}$ revealed that CTLs of gzmAB ${ }^{-1-}$ mice, which expressed only GzmK and very limited quantities of GzmC, showed similar viral clearance capacity as did wild-type (WT) controls. This indicates that GzmK has a critical role in the elimination of the influenza virus. However, how GzmK inhibits viral replication remains unclear.

GzmK is the alternate tryptase among all Gzms, linking closely with the tryptase GzmA on the same chromosome in humans and mice. GzmK is expressed to a great degree in CD56 ${ }^{\text {bright }}$ natural killer (NK) cells, $\mathrm{CD}^{+}{ }^{+} \mathrm{CD}^{+}{ }^{+}$and $\mathrm{CD} 4^{+}$ cytotoxic $\mathrm{T}$ cells, and $\gamma \delta \mathrm{T}$ cells. ${ }^{9}$ We have previously demonstrated that GzmK targets the SET complex to initiate caspase-independent cell death. ${ }^{10,11} \mathrm{GzmK}$ can induce the rapid generation of reactive oxygen species through a Biddependent pathway. ${ }^{12}$ GzmK also cleaves the valosincontaining protein (VCP) to destroy the ER-associated degradation (ERAD) pathway, resulting in an ER stress of the target cell. ${ }^{13}$ We also found that GzmK proteolyzes the tumor suppressor p53 to generate three pro-apoptotic segments that potentially increase elimination of target tumor cells. ${ }^{14}$ In this study, we showed that GzmK can cleave the importin $\alpha 1 / \beta$ dimer and disrupt transportation of viral NP to the nucleus, resulting in elimination of influenza virus in vivo.

\section{Results}

GzmK blockade aggravates influenza virus infection. Harari et al. $^{15}$ demonstrated that influenza $A$ virus-specific $\mathrm{CD}^{+} \mathrm{T}$ cells possess a profile of perforin ${ }^{-} \mathrm{GzmB}^{-} \mathrm{GzmA}^{+/-}$ $\mathrm{GzmK}^{+}$. In $\mathrm{CD}^{+}, \mathrm{CTL}$ cells of $\mathrm{gzmAB}^{-1-}$ mice, which express only GzmK and very limited amounts of GzmC, exhibited effective clearance of influenza virus. ${ }^{8}$ These

\footnotetext{
${ }^{1}$ CAS Key Laboratory of Infection and Immunity and Center for Infection and Immunity, Institute of Biophysics, Chinese Academy of Sciences, Beijing, China and ${ }^{2}$ Fukushimura Hospital, Choju Medical Institute, Toyohashi, Japan

*Corresponding author: Z Fan, Center for Infection and Immunity, Institute of Biophysics, Chinese Academy of Sciences, Beijing 100101, China. Tel: + 86 10 64888457; Fax: + 8610 64871293; E-mail: fanz@moon.ibp.ac.cn

Keywords: granzyme K; importin $\alpha 1$; importin $\beta$; influenza A virus; substrate

Abbreviations: Ad, replication-deficient adenovirus type V; ARM, armadillo; ERG, energy regeneration system; Gzm, granzyme; HEAT, Huntington, elongation factor 3 ,

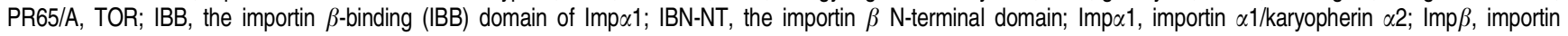
$\beta /$ karyopherin $\beta 1$; rlmp, recombinant importin; KI, GzmK inhibitor; NP, nucleoprotein; WT, wild-type

Received 13.7.11; revised 29.9.11; accepted 02.11.11; Edited by S Kornbluth; published online 02.12.11
} 
reports suggest that GzmK has a critical role in the host's defense against influenza virus infection. To determine the role of GzmK in the elimination of influenza virus in vivo, Balb/c mice (4-6-weeks old) were intravenously injected with GzmK-specific inhibitor (KI) to inhibit endogenous GzmK before viral challenge. We have previously showed that the GzmK inhibitor Ac-YRFK-CMK can specifically block enzymatic activity of GzmK. ${ }^{13}$ Mice were then intranasally infected with the Flu A/WSN/33 (H1N1) (10² PFU/mouse) virus. KI-pretreated mice showed significantly higher viral loads in their lungs than did PBS controls at both 3 days and 6 days post infection (p.i.) (Figure 1a). At 3 d.p.i., viruspositive cells were found mainly in the bronchial epithelium in PBS control mice, whereas mice treated with the GzmK inhibitor also showed infected regions in alveolar epithelial cells around the bronchia (Figure 1b). At 6 d.p.i., infections of PBS control mice appeared to be restricted to the alveolar epithelium and showed much weaker viral protein staining intensity relative to $\mathrm{KI}$-pretreated mice. In contrast, $\mathrm{KI}$-pretreated mice showed heavy staining on most bronchial cells (Figure 1b). We further confirmed the above findings through detection of the intensity of vRNA in infected cells through in situ hybridization assay. Lung sections of 6 d.p.i. mice were detected for vRNA for the M2 protein. KI-pretreated mice showed more viral RNA-hybridized cells and more hybrid points in infected cells than did PBS control mice (Figure 1c). These results indicate that GzmK has a critical role in the elimination of influenza $A$ virus.

\section{Attenuation of LAK cell-mediated clearance of influenza virus by GzmK inhibition. We further explored whether} lymphokine-activated killer (LAK) cells participate in clearance of the influenza virus. LAK cells were obtained from PBMC cells (healthy donors) with IL-2 (1000 Units/ml) stimulation. We used a luciferase reporter system to detect the replication of influenza A virus as described previously. ${ }^{16}$ The reporter plasmid pPollNSluc was transfected into human alveolar epithelial cell line A549 cells $12 \mathrm{~h}$ before infection along with an intrinsic control plasmid pRL-SV40. The above treated cells were then infected with Flu A/WSN/33 (H1N1) and incubated with LAK cells with or without $\mathrm{KI}$ at an $\mathrm{E} / \mathrm{T}$ ratio of $1: 1$ for $24 \mathrm{~h}$. Viral replication in infected cells was analyzed through a dual luciferase assay. Infected A549 cells were all alive at this point in time (data not shown). LAK cells repressed influenza virus replication by $53.4 \%$ (Figure $2 a$ ). In contrast, LAK cells with GzmK inhibition elevated replication over $49.0 \%$ relative to LAK cell-treated target cells. To further verify the inhibitory role of GzmK in influenza virus replication, we simplified the factors for influenza virus replication, assuming that only viral polymerase and NP protein (Pol $+\mathrm{NP})$ were necessary for VRNA amplification. The reporter system and experimental procedure were the same as those used for cells infected with Flu A/WSN/33 (H1N1). As expected, LAK cells significantly inhibited the replication of vRNA by $81.7 \%$, whereas LAK cells with GzmK inhibition rescued repression by $59.0 \%$ (Figure $2 b$ ). a

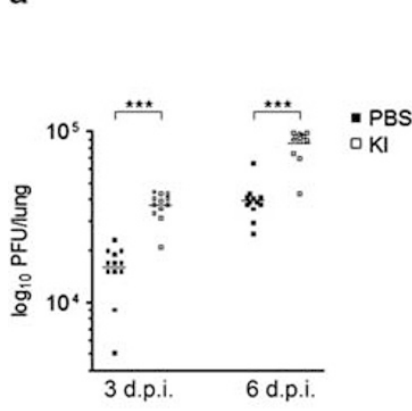

b

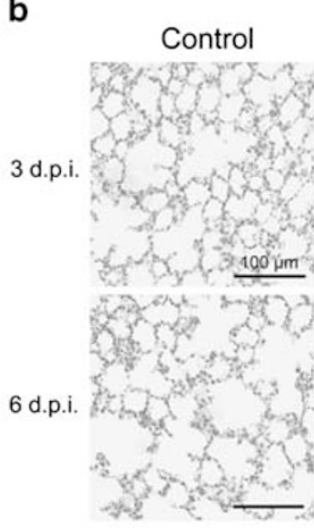

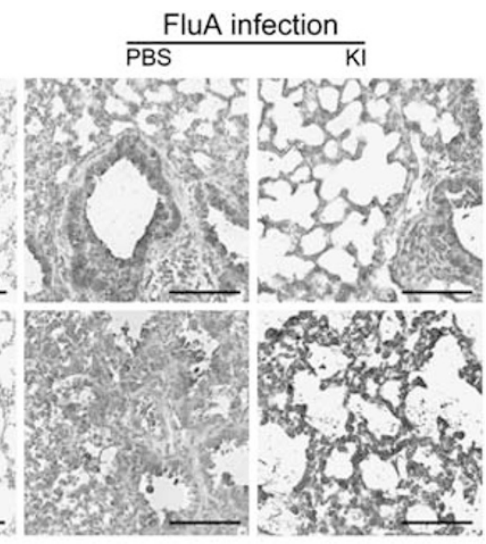
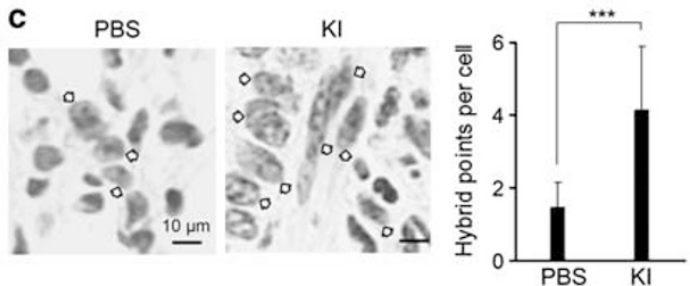

Figure 1 GzmK blockage aggravates influenza virus infection. (a) The GzmK inhibitor elevates viral load in infected mouse lungs. Balb/c mice were intravenously injected with KI or PBS 1 day before virus infection. Mice were then infected intranasally with Flu A/WSN/33 (H1N1). Viral titers in the infected lungs were quantified using plaque assay at 3 days post-injection (d.p.i.) and 6 d.p.i. Kl: GzmK inhibitor. ${ }^{* \star *} P<0.0001$. (b) GzmK inhibition aggravates viral infection. The viral protein M2 was detected in the infected lungs by immunohistochemistry (IHC) at 3 and 6 d.p.i. The M2 protein of Flu A/WSN/33(H1N1) was stained blue with NBT/BCIP, and the nucleus was stained red with nuclear fast red. Control mouse lungs were visualized as a negative control. (c) GzmK inhibition rescues viral RNA replication in the infected mouse lungs. Viral M2 RNA was detected by ISH (in situ hybridization) IHC assay. Hybrid points were stained through the DAB system, and the nucleus was stained by hematoxylin. The arrows indicate cells with hybrid points in nuclei. ${ }^{\star \star \star} P<0.0001$. The color reproduction of this figure is available at the Cell Death and Differentiation journal online 
GzmK associates with importin $\alpha 1$. An inactive GzmK mutant (S-AGzmK) was immobilized with Affi-Gel10 resin and passed through $\mathrm{K} 562$ cell lysates for affinity chromatography. The S-AGzmK column was eluted with $\mathrm{NaCl}$ concentration gradients. After elution with $500 \mathrm{mM}$ $\mathrm{NaCl}$, the eluted fractions were resolved on SDS-PAGE, followed by silver staining (Figure 3a). The six major bands
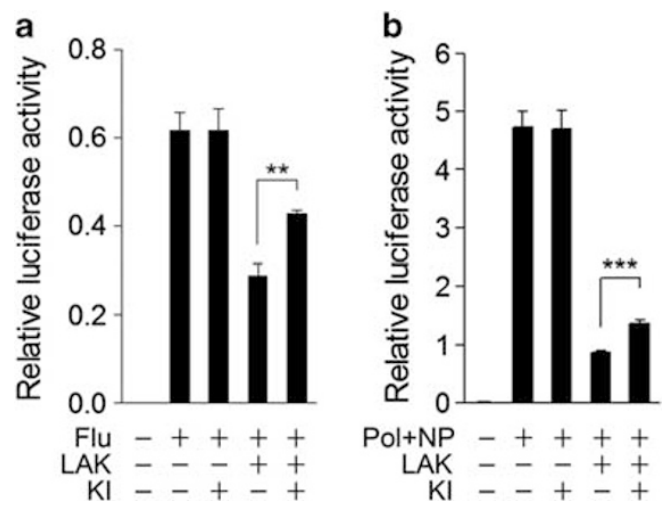

Figure 2 GzmK inhibition attenuates LAK cell-mediated clearance of influenza virus. (a) The GzmK inhibitor significantly impedes LAK cell-mediated viral clearance. A549 cells were transfected with influenza A luciferase plasmid pPollNS-luc and an intrinsic control plasmid pRL-SV40. After $12 \mathrm{~h}$, treated cells were infected with Flu A/WSN/33 ( $\mathrm{H} 1 \mathrm{~N} 1)$ at an MOI of $10^{-2}$ for $2 \mathrm{~h}$. The infected $\mathrm{A} 549$ cells were incubated with IL-2-activated LAK cells at an E/T ratio of $1: 1$ followed by luciferase assay. For GzmK inhibition, LAK cells were pretreated with KI for $2 \mathrm{~h}$. ${ }^{\star *} P<0.001$. (b) GzmK inhibition facilitates LAK cell-mediated replication of the influenza virus. A549 cells were transfected with influenza A polymerase (PB1, PB2, and PA) and NP plasmids (Pol + NP), the luciferase plasmid pPoll-NS-luc, and the control plasmid pRL-SV40, followed by the above assay. ${ }^{\star \star \star} P<0.0001$ were eluted and cut out for mass spectrometry. These proteins were identified as VCP, importin $\alpha 1$, tubulin $\beta$, OLA1, SET, and pp32. We have previously demonstrated that VCP and SET are physiological substrates of GzmK and that pp32 associates with GzmK in the SET complex. ${ }^{10,11}$ Interestingly, tubulin $\beta$ was also identified as a physiological substrate of GzmK by the Bovenschen et al. ${ }^{17}$ OLA1 is a kind of ATPase that was reported to suppress cellular antioxidant response. ${ }^{18}$ Importin $\alpha 1$, a member of the karyopherin $\alpha$ family, acts as a component of the nuclear transport complex to transport protein cargos between the cytoplasm and the nucleus. ${ }^{19}$ Intriguingly, host cell importin $\alpha 1$ was reported to bind the NP and PB2 of influenza A virus and to import them into the nucleus for replication of the viral genome. ${ }^{2,20} \mathrm{We}$ next decided to determine whether GzmK would associate with importin $\alpha 1$ under physiological conditions. S-AGzmK could precipitate native importin $\alpha 1$ in K562 cell lysates (Figure 3b). Importin $\alpha 1$ also precipitated S-AGzmK from S-AGzmK-incubated lysates. Similar results were obtained using HeLa cell lysates (data not shown). IgG used as a control did not pull down either importin $\alpha 1$ or S-AGzmK. To confirm the direct interaction of GzmK with importin $\alpha 1$, recombinant importin $\alpha 1 \quad(\mathrm{r} \mid \mathrm{mp} \alpha 1)$ was incubated with S-AGzmK for co-immunoprecipitation assay. S-AGzmK co-precipitated $\mathrm{rlmp} \alpha 1$ and vice versa (Figure $3 \mathrm{c}$ ), whereas control IgG and rGST had no effect. Therefore, it was concluded that S-AGzmK binds directly to importin $\alpha 1$ in its recombinant and native forms.

Importin $\alpha \mathbf{1}$ is a physiological substrate of GzmK. To determine whether importin $\alpha 1$ is cleaved by GzmK, rlmp $\alpha 1$ $(0.5 \mu \mathrm{M})$ was incubated with different concentrations of a

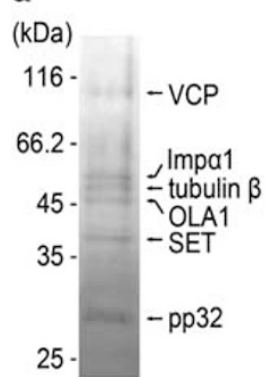

b

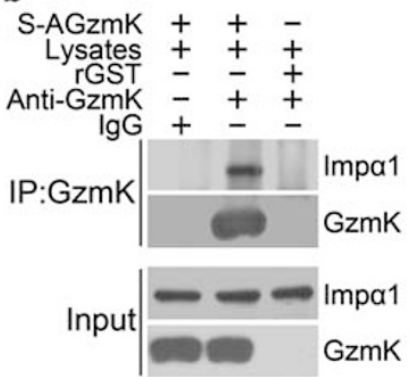

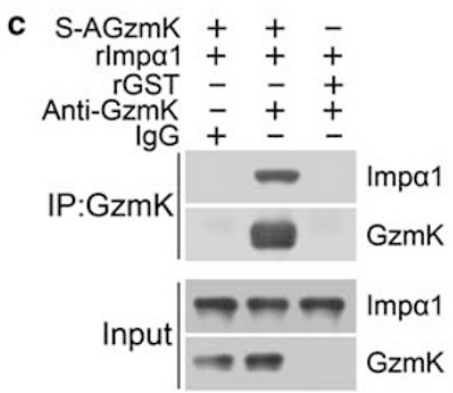

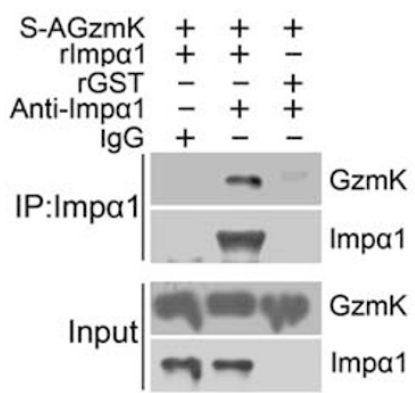

Figure 3 GzmK associates with Importin $\alpha 1$. (a) Importin $\alpha 1$ (Imp $\alpha 1$ ) was eluted with the SET complex. The eluted bands were identified by mass spectrometry. (b) GzmK interacts with native importin $\alpha 1$. In all, $10 \mu \mathrm{g}$ S-AGzmK was incubated with K562 cell lysates followed by co-immunoprecipitation with anti-GzmK antibody (left panel) or antiimportin $\alpha 1 \mathrm{mAb}$ (right). (c) GzmK binds directly to recombinant importin $\alpha 1$ (rlmp $\alpha 1$ ). In all, $10 \mu \mathrm{g}$ S-AGzmK and $10 \mu \mathrm{g}$ rlmp $\alpha 1$ were incubated and co-immunoprecipitation assay was performed as described above. IgG served as a negative antibody control, and rGST was used as a negative protein control. IP: immunoprecipitation 


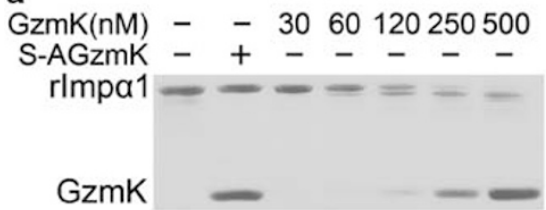

C

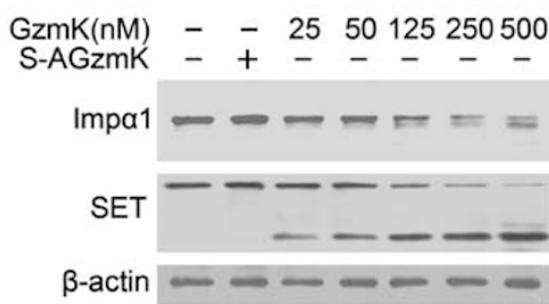

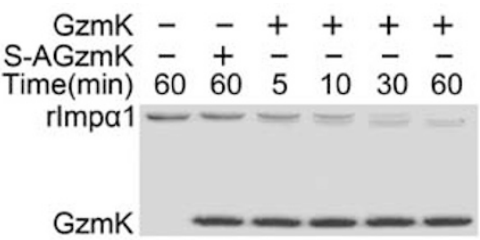
$\begin{array}{rrrrrrr}\text { GzmK } & - & - & + & + & + & + \\ \text { S-AGzmK } & - & + & \overline{+} & - & - & - \\ \text { Time(min) } & 60 & 60 & 5 & 10 & 30 & 60\end{array}$

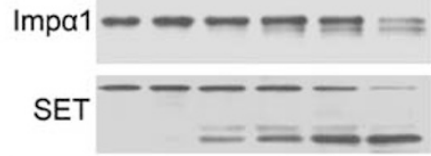

$\beta$-actin ---

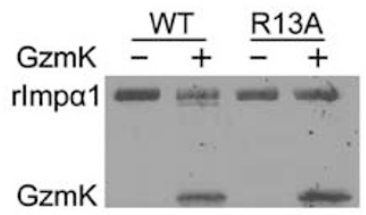

d

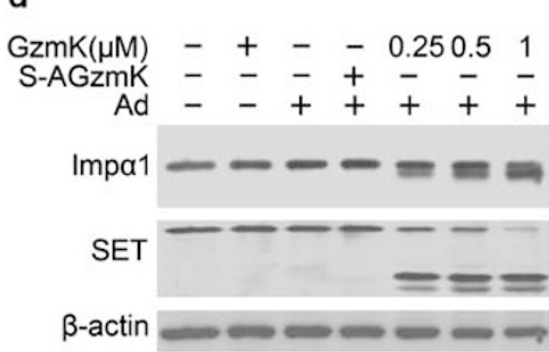

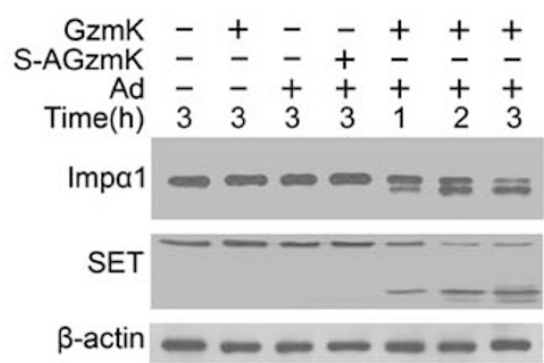

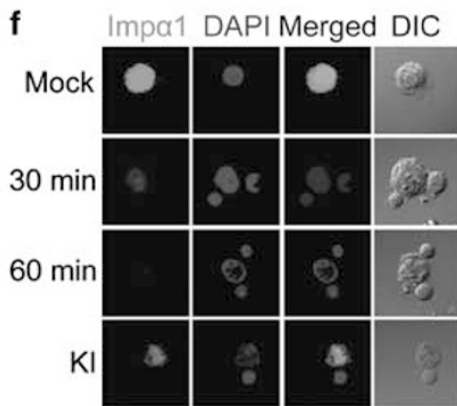

Figure 4 Importin $\alpha 1$ is a physiological substrate for GzmK. (a) GzmK directly cleaves rlmp $\alpha 1$ in a dose- and time-dependent manner. rlmp $\alpha 1$ (0.5 $\mu \mathrm{M})$ was incubated with different doses of GzmK for $1 \mathrm{~h}$ or with $0.5 \mu \mathrm{M}$ GzmK for the indicated times at $37^{\circ} \mathrm{C}$. The reactions were resolved by SDS-PAGE and visualized by Coomassie brilliant blue staining. S-AGzmK served as a negative enzyme control. (b) GzmK cuts importin $\alpha 1$ at Arg ${ }^{13}$. Wild-type (WT) and R13A-Imp $\alpha 1$ were treated with $0.5 \mu \mathrm{M}$ GzmK followed by Coomassie staining. (c) GzmK cleaves native importin $\alpha 1$ in a dose- and time-dependent manner. K562 cell lysates $\left(2 \times 10^{5}\right.$ equivalents) were treated with different concentrations of GzmK for $1 \mathrm{~h}$ or with $0.5 \mu \mathrm{M} \mathrm{GzmK}$ for the indicated times at $37^{\circ} \mathrm{C}$. (d) GzmK degrades importin $\alpha 1$ in GzmK-loaded intact cells. In all, $2 \times 10^{5}$ Jurkat cells were incubated with different doses of GzmK for $3 \mathrm{~h}$ or with $1 \mu \mathrm{M} \mathrm{GzmK}$ for the indicated times at $37^{\circ} \mathrm{C}$ in the presence of replication-deficient adenovirus type V (Ad) (100 PFU/ml). SET was used as a positive control. $\beta$-Actin remained unchanged as a loading control. (e and f) GzmK degrades importin $\alpha 1$ in LAK cell-mediated cytolysis. K562 cells expressing Imp $\alpha 1$-Myc were incubated with IL-2activated LAK cells at the indicated E/T ratios followed by immunoblotting. The GzmK known substrate Bid was detected as a positive control. GFP was expressed in K562 cells and visualized as a loading control (panel e). Importin $\alpha 1$ was visualized with anti-Myc antibody (red) using the immunofluorescence assay (panel f). Nuclei were stained with DAPI (blue). DIC: differential interference contrast. The color reproduction of this figure is available at the Cell Death and Differentiation journal online

GzmK at $37^{\circ} \mathrm{C}$ for $1 \mathrm{~h}$ or with $0.5 \mu \mathrm{M}$ GzmK for 5-60 min. rImp $\alpha 1$ began to be degraded at a low concentration of $60 \mathrm{nM}$ GzmK (Figure 4a, left panel). rImp $\alpha 1$ was completely processed at $0.25 \mu \mathrm{M}$ GzmK. By $5 \mathrm{~min}$, rImp $\alpha 1$ began to be proteolyzed and was completely processed after $30 \mathrm{~min}$ (Figure 4a, right panel). rlmp $\alpha 1$ was not cleaved by S-AGzmK. Thus, it was concluded that $\operatorname{rmp} \alpha 1$ degradation requires the enzymatic activity of GzmK. We further identified the cleavage site by using site-directed mutagenesis. The Arg13Ala mutant (R13A-Imp $\alpha 1$ ) was not cleaved by GzmK (Figure 4b). Therefore, GzmK cleaved importin $\alpha 1$ after Arg $^{13}$ at the $\mathrm{N}$ terminus to generate a truncated form (tImp $\alpha 1)$.

To further confirm that GzmK cleaves native importin $\alpha 1$ in mammalian cells, $\mathrm{K} 562$ cell lysates $\left(2 \times 10^{5}\right.$ equivalent) were incubated with different concentrations of GzmK at $37^{\circ} \mathrm{C}$ for 60 min. Native importin $\alpha 1$ in cell lysates was degraded by GzmK in a dose-dependent manner, whereas the inactive S-AGzmK exerted no effect (Figure 4c, left panel). The SET protein, a substrate of GzmK, served as a positive cleavage control and $\beta$-actin served as a negative control. Moreover,
Imp $\alpha 1$ began to be cleaved by GzmK by $10 \mathrm{~min}$ in a timedependent manner (Figure 4c, right panel). To further confirm that importin $\alpha 1$ processing is physiologically relevant, K562 cells were treated with different concentrations of GzmK for $3 \mathrm{~h}$ or with $1 \mu \mathrm{M}$ GzmK for $1-3 \mathrm{~h}$ in the presence of replicationdeficient adenovirus type $\mathrm{V}(\mathrm{Ad})$. As expected, importin $\alpha 1$ of target cells was cleaved in a dose- and time-dependent manner (Figure 4d). GzmK and Ad alone or S-AGzmK plus Ad were not able to degrade importin $\alpha 1$. SET cleavage also served as a positive control and $\beta$-actin remained unchangeable as a good loading control. Similar results were found using HeLa cells (data not shown).

GzmK inhibition blocks degradation of importin $\alpha 1$ in LAK cell-mediated cytolysis. We further determined whether GzmK could cause degradation of importin $\alpha 1$ in LAK cell-mediated cytotoxicity. The Imp $\alpha 1-M y c$ protein was overexpressed in K562 cells using an MSCV-IRES-GFP retroviral system. Positive cells expressing Imp $\alpha 1-M y c$ were sorted by a GFP marker through an FACS sorter. K562 cells 
with the Impa1-Myc expression were incubated with IL-2activated LAK cells at E/T ratios of $5: 1$ and $10: 1$ followed by immunoblotting. For GzmK inhibition, LAK cells were pretreated with the GzmK inhibitor. Importin $\alpha 1$ was degraded at an E/T ratio of $5: 1$ and almost proteolyzed at an $E / T$ ratio of $10: 1$ (Figure 4e). Remarkably, the GzmK inhibitor inhibited degradation of importin $\alpha 1$ at both $E / T$ ratios. Bid was probed as a positive control and GFP served as a target cell loading control. These results were also confirmed by confocal microscopy (Figure 4f). Imp $\alpha 1$-Myc-expressing K562 cells were cocultured with LAK cells for 30 and $60 \mathrm{~min}$ and stained with anti-Myc antibody and DAPI. Importin $\alpha 1$ of K562 cells was degraded after as little as $30 \mathrm{~min}$ and completely processed at $60 \mathrm{~min}$. GzmK inhibition blocked degradation of importin $\alpha 1$ during attack by LAK cells. Taken together, importin $\alpha 1$ is a physiological substrate for GzmK.

GzmK also degrades the other transport partner importin $\boldsymbol{\beta}$. In the classical nuclear import pathway, importin $\alpha 1$ first recognizes its cytoplasmic cargos by their classical nuclear localization sequences (NLSs), and then associates with importin $\beta$ (karyopherin $\beta 1$ ) to form a ternary import complex for transportation. ${ }^{21}$ In this manner, importin $\beta$ acts as a transport partner for importin $\alpha 1$. We decided to look at whether GzmK could degrade importin $\beta$ in host cells. Recombinant importin $\beta(\operatorname{rlmp} \beta)(0.5 \mu \mathrm{M})$ was incubated with different doses of GzmK. $\operatorname{rmp} \beta$ began to be cleaved at a very low concentration of $10 \mathrm{nM}$ GzmK and was completely processed at $0.2 \mu \mathrm{M}$ GzmK in a dose-dependent manner (Figure 5a, left panel). GzmK also proteolyzed $\operatorname{rlmp} \beta$ in a time-dependent manner (Figure $5 \mathrm{a}$, right panel). Inactive S-AGzmK had no effect. The cleavage site was identified at $\mathrm{Arg}^{710}$ of the $\mathrm{C}$ terminus through site-directed mutagenesis (Figure $5 \mathrm{~b})$. K562 cell lysates $\left(2 \times 10^{5}\right.$ equivalent) were incubated with different concentrations of GzmK for $1 \mathrm{~h}$ or with $0.5 \mu \mathrm{M}$ GzmK for the indicated lengths of time. Native importin $\beta$ was degraded by GzmK in a dose- and timedependent manner (Figure 5c). The GzmK substrate SET served as a positive control and $\beta$-actin was used as a negative control. S-AGzmK displayed no effect. Moreover, importin $\beta$ was degraded in GzmK-loaded intact K562 cells (Figure $5 d$ ). Meanwhile, importin $\alpha 1$ and SET were a

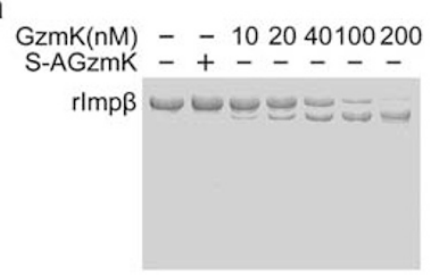

C

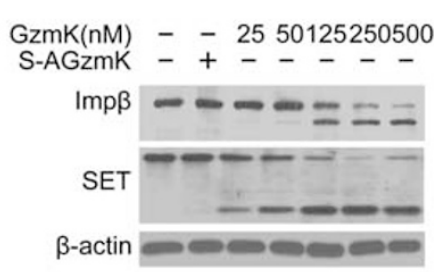

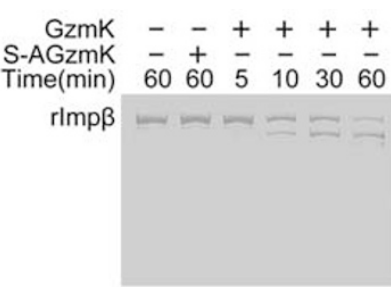

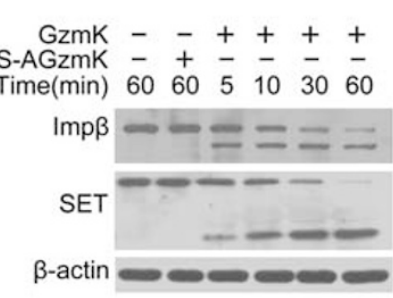

b

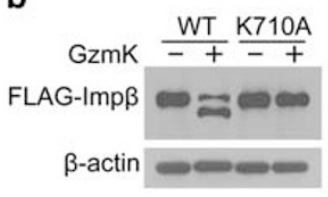

d

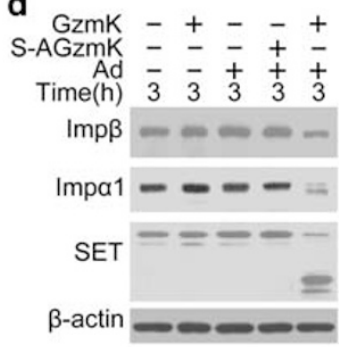

e

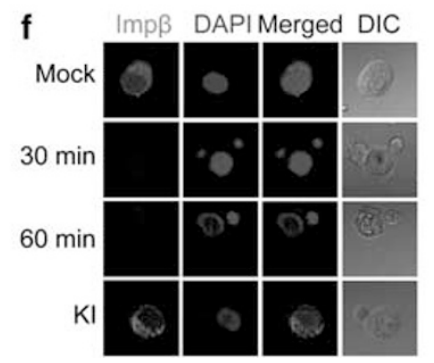

Figure 5 GzmK also degrades the transport partner importin $\beta$ after Lys ${ }^{710}$. (a) GzmK directly cleaves recombinant importin $\beta$ (rlmp $\beta$ ) in a dose- and time-dependent manner. rImp $\beta(0.5 \mu \mathrm{M})$ was incubated with different concentrations of $\mathrm{GzmK}$ for $1 \mathrm{~h}$ or with $10 \mathrm{nM} \mathrm{GzmK}$ for the indicated times at $37^{\circ} \mathrm{C}$ followed by Coomassie staining. (b) GzmK cleaves importin $\beta$ at Lys ${ }^{710}$. Wild-type (WT) and FLAG-K710A-Imp $\beta$ were transiently expressed in 293 T cells for $48 \mathrm{~h}$. Cell lysates $\left(2 \times 10^{5}\right.$ equivalents) were treated with $0.5 \mu \mathrm{M} \mathrm{GzmK}$ for $15 \mathrm{~min}$ and detected by anti-FLAG mAb. $\beta$-Actin was used as a negative control. (c) GzmK proteolyzes the native importin $\beta$ of cell lysates in a dose- and time-dependent manner. K562 cell lysates $\left(2 \times 10^{5}\right.$ equivalents) were incubated with different concentrations of GzmK for $1 \mathrm{~h}$ or with $0.5 \mu \mathrm{M} \mathrm{GzmK}$ for the indicated times at $37^{\circ} \mathrm{C}$. SET was probed as a positive control and $\beta$-actin was used as a negative control. (d) GzmK cleaves importin $\beta$ in GzmK-loaded intact cells. Jurkat cells were incubated with $1 \mu \mathrm{M} \mathrm{GzmK}$ and $\mathrm{Ad}(100 \mathrm{PFU} / \mathrm{ml})$ for $3 \mathrm{~h}$ at $37^{\circ} \mathrm{C}$ and probed for importin $\beta$, importin $\alpha 1$, SET, and $\beta$-actin. (e and f) GzmK cuts importin $\beta$ during the LAK cellmediated killing process. K562 cells expressing FLAG-Imp $\beta$ were incubated with IL-2-activated LAK cells at the indicated E/T ratios followed by immunoblotting. GFP was specifically expressed in K562 cells and visualized as a loading control (panel e). Importin $\beta$ was probed with anti-FLAG mAb (red) using immunofluorescence assay (panel f). Nuclei were stained with DAPI (blue). DIC: differential interference contrast. The color reproduction of this figure is available at the Cell Death and Differentiation journal online 
probed as positive controls. $\beta$-Actin was unchanged as a good loading control.

We next detected degradation of importin $\beta$ in LAK cellattacked target cells. FLAG-Imp $\beta$-expressing K562 cells were incubated with IL-2-activated LAK cells at E/T ratios of $5: 1$ and $10: 1$. We found that importin $\beta$ was degraded at both $\mathrm{E} / \mathrm{T}$ ratios (Figure $5 \mathrm{e}$ ). GzmK inhibition suppressed the degradation of importin $\beta$. Similar results were confirmed by confocal assay (Figure 5f). Within $30 \mathrm{~min}$, FLAG-tagged importin $\beta$ was almost degraded by LAK cells, whereas this degradation could be impeded by the GzmK inhibitor (Figure 5f). Therefore, importin $\beta$ is also a physiological substrate for GzmK.

GzmK-truncated importin $\alpha 1(\operatorname{tImp} \alpha 1)$ and importin $\beta$ $(\operatorname{tImp} \beta)$ destroy their association. GzmK cleaved importin $\alpha 1$ after $\mathrm{Arg}^{13}$ in the importin $\beta$-binding (IBB) domain that mediates the interaction of importin $\alpha 1$ with importin $\beta$ (Figure $6 \mathrm{a}$ ). $\mathrm{Arg}^{13}$ is the only strictly conserved residue in the IBB domain of all the importin $\alpha$ homologs, making it critical to interactions with importin $\beta$ and formation of the transport complex. ${ }^{22}$ Thus, we determined whether depletion of the $\mathrm{N}$-terminal moiety of importin $\alpha 1$ by GzmK would impact interactions between importin $\alpha 1$ and importin $\beta$. Here, $293 \mathrm{~T}$ cells were transiently cotransfected with Myctagged importin $\alpha 1$ (Imp $\alpha 1-\mathrm{Myc})$ or its GzmK-truncated fragment (tImp $\alpha 1-\mathrm{Myc})$ and FLAG-tagged importin $\beta$ $($ FLAG-Imp $\beta$ ) for $48 \mathrm{~h}$, followed by the co-immunoprecipitation assay. tImp $\alpha 1-$ Myc was not precipitated by the anti-FLAG antibody (Figure $6 \mathrm{~b}$ ), but Imp $\alpha 1-\mathrm{Myc}$ was precipitated as a good interaction control. The anti-FLAG antibody failed to pull down any protein in $293 \mathrm{~T}$ cells
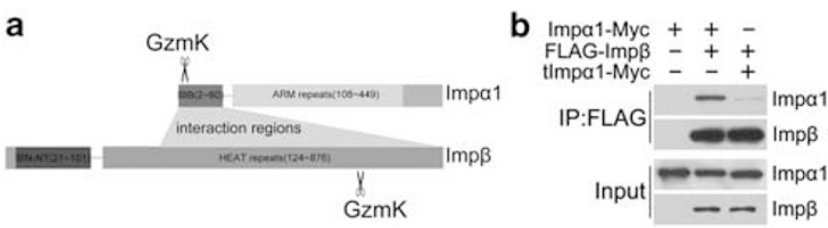

C
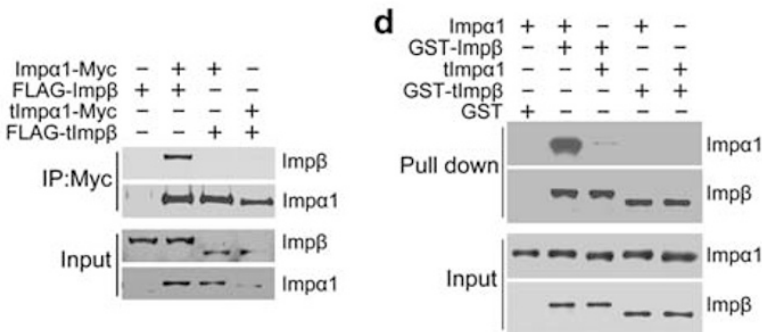

Figure 6 GzmK-truncated importin $\alpha 1(\operatorname{tmp} \alpha 1)$ and importin $\beta(\operatorname{tmp} \beta)$ destroy their association. (a) A schematic map shows the regions of interaction between importin $\alpha 1$ and importin $\beta$. IBB: The importin $\beta$-binding (IBB) domain of Imp $\alpha 1$; ARM: armadillo; IBN-NT: the importin $\beta$ N-terminal domain; HEAT: Huntington, elongation factor $3, P R 65 / A, T O R$. (b and $\mathbf{c}$ ) $\operatorname{timp} \alpha 1$ or $\operatorname{timp} \beta$ disrupts the interaction. 293 T cells were co-transfected with full-length or $\operatorname{timp} \alpha 1$ and $\operatorname{tImp} \beta$ for $48 \mathrm{~h}$. Their interactions were detected by co-immunoprecipitation assay. (d) Similar results were confirmed by GST pull-down assay. GST-Imp $\beta$ and GST-tImp $\beta$ were incubated with recombinant Imp $\alpha 1$ or tImp $\alpha 1$ overnight at $4^{\circ} \mathrm{C}$ and further incubated with GST beads for $2 \mathrm{~h}$. Protein interactions were assayed by immunoblotting. rGST served as a negative control expressing Imp $\alpha 1-$ Myc alone. Similarly, 293 T cells were transiently cotransfected with FLAG-tagged importin $\beta$ (FLAG-Imp $\beta$ ) or its GzmK-truncated fragment (FLAG$\operatorname{tImp} \beta)$, as well as $\operatorname{Imp} \alpha 1-\mathrm{Myc}$ or tImp $\alpha 1-$ Myc for immunoprecipitation. As expected, FLAG-tImp $\beta$ was not pulled down by the anti-Myc antibody (Figure $6 \mathrm{c}$ ), whereas FLAG-Imp $\beta$ was precipitated by this antibody. Notably, tImp $\alpha 1-\mathrm{Myc}$ and FLAG-tImp $\beta$ did not interact (Figure $6 c$ ). Similar results were obtained using GST-tagged importin $\beta($ GST-Imp $\beta)$ or its GzmK-truncated fragment (GST-tImp $\beta$ ) (Figure 6d). These results indicate that the interaction between $\operatorname{Imp} \alpha 1$ and $\operatorname{Imp} \beta$ was disrupted after cleavage by GzmK.

tImp $\alpha 1$ or $\operatorname{tImp} \beta$ abolishes NP transportation resulting in inhibition of viral replication. As shown above, GzmKtruncated $\operatorname{tImp} \alpha 1$ and $\operatorname{tImp} \beta$ lost their ability to interact with their full-length forms. We next tested whether tImp $\alpha 1$ or $\operatorname{timp} \beta$ could affect transportation of the viral NP protein. First, 293 T cells were cotransfected with the HA-tagged NP (HA-NP) protein and either Imp $\alpha 1-M y c$ or tImp $\alpha 1-M y c$. We demonstrated that $\operatorname{tImp} \alpha 1$ could bind to the NP protein, as did the full-length importin $\alpha 1$ (Figure 7a). To determine effects of $\operatorname{tImp} \alpha 1$ and $\operatorname{tImp} \beta$ on the transportation of NP protein, HeLa cells were precultured for $18 \mathrm{~h}$, penetrated with $40 \mu \mathrm{g} / \mathrm{ml}$ digitonin and then incubated with the Myc-NP protein. Transportation factors for this in vitro transport assay included Ran-GDP and NTF/p10, the indicated combinations of full-length and truncated importin proteins, and an energy regeneration system (ERG). Transport of NP was visualized by immunofluorescence with anti-Myc antibody. Full-length importin $\alpha 1$ and importin $\beta$ transported the NP protein into the nucleus (Figure $7 \mathrm{~b}$ ). However, tImp $\alpha 1$ with importin $\beta, \operatorname{tImp} \beta$ with importin $\alpha 1$, and $\operatorname{tImp} \alpha 1$ with $\operatorname{tImp} \beta$ were not. In the above system, we substituted GzmK-treated cell lysates for rlmp proteins to obtain similar results (Figure 7c). GzmK treatment blocked nuclear transportation of the NP protein. As a negative control, it was established that the NP protein would undergo no transportation without ERG.

The NP protein forms the vRNP complex with influenza virus vRNA for viral replication. ${ }^{23}$ We supposed that blocking NP transportation might lead to inhibition of viral replication. First, we established a stable 293 T-cell line with importin $\alpha 1$ and importin $\beta$ double depletion (Figure 7d). WT 293 T and importin $\alpha 1$ and importin $\beta$ double-depletion cell lines were cotransfected with plasmids for expression of Pol + NP and pPollNSluc. pRL-SV40 served as an intrinsic transfection control. Double knockdown of importin $\alpha 1$ and importin $\beta$ significantly suppressed vRNA polymerase activity (Figure 7d). To further confirm the effects of importin $\alpha 1$ and importin $\beta$ on viral replication, we overexpressed full-length importin $\alpha 1$ and importin $\beta, \operatorname{tImp} \alpha 1$ with importin $\beta, \operatorname{timp} \beta$ with importin $\alpha 1$, and $\operatorname{tmp} \alpha 1$ with $\operatorname{tImp} \beta$ in the $293 \mathrm{~T}$-cell line with double depletion of importin $\alpha 1$ and importin $\beta$. Transfection of importin $\alpha 1$ and importin $\beta$ rescued polymerase activity (Figure 7e). As expected, tImp $\alpha 1$ with importin $\beta, \operatorname{timp} \beta$ with importin $\alpha 1$, and $\operatorname{tImp} \alpha 1$ with $\operatorname{tmp} \beta$ transfection had no effect. These results indicate that truncation of either importin $\alpha$ or importin $\beta$ disables transport of the NP protein, leading to inhibition of viral replication. 


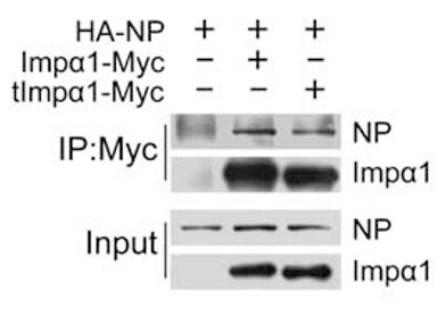

d

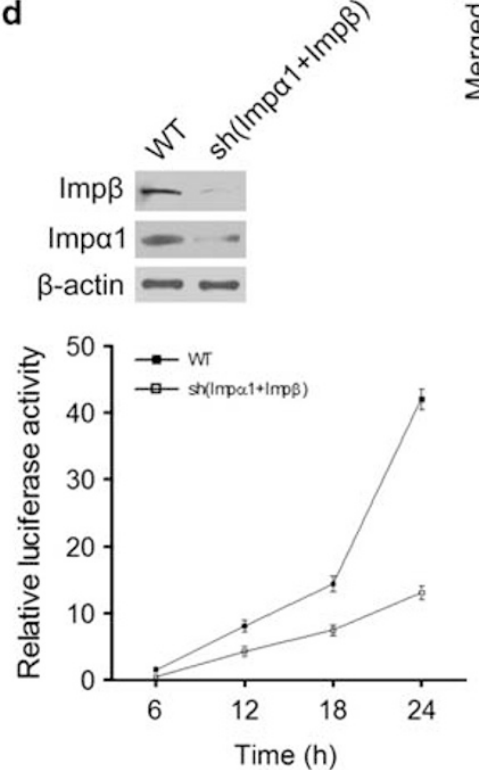

b

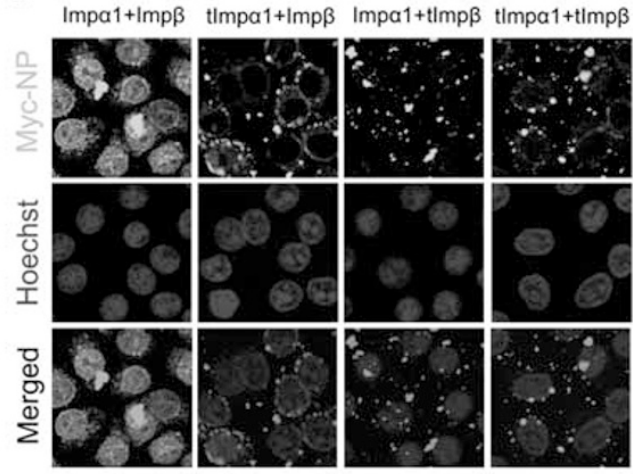

C

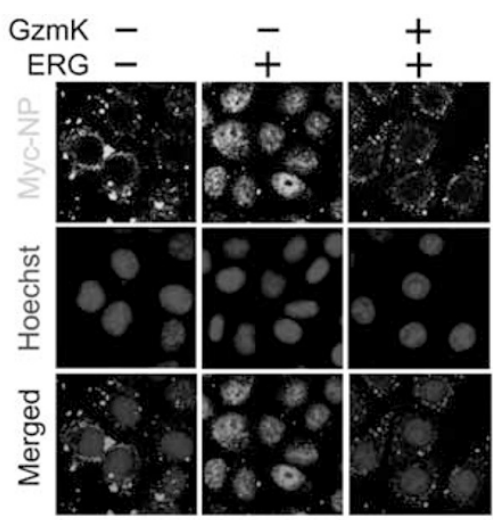

\section{e}

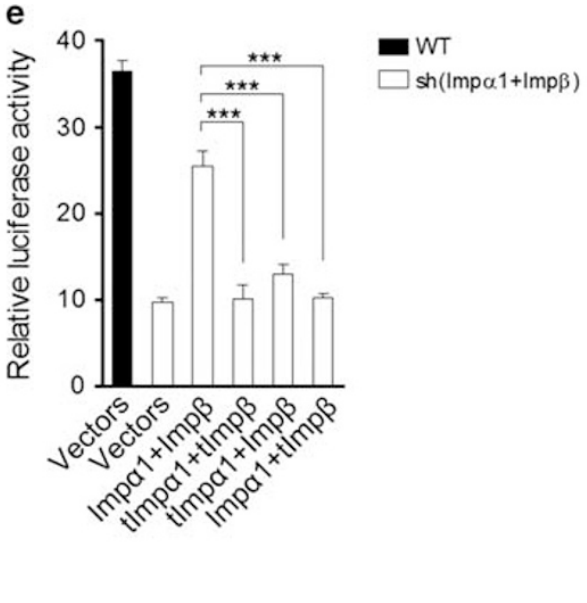

Figure 7 Both $\operatorname{tmp} \alpha 1$ and tImp $\beta$ abolish viral NP transportation to disable replication of influenza virus. (a) Importin $\alpha 1$ and tImp $\alpha 1$ bind to viral NP. 293 T cells were co-transfected with Imp $\alpha 1$-Myc or tImp $\alpha 1$-Myc plus HA-NP for $48 \mathrm{~h}$. Anti-Myc antibody was used to immunoprecipitate the cell lysates, which were then immunoblotted with anti-HA antibody. (b) $\operatorname{tmp} \alpha 1$ and $\operatorname{tImp} \beta$ disrupt the transportation of NP into the nucleus. Digitonin-pre-permeabilized HeLa cells were incubated with recombinant Myc-NP protein in the presence of importin $\alpha 1$ or $\operatorname{tImp} \alpha 1$ and $\operatorname{Imp} \beta$ or $\operatorname{tImp} \beta$, as well as other import factors and the energy regeneration system (ERG). Importation of recombinant Myc-NP was detected by anti-Myc antibody through immunofluorescence. (c) GzmK-treated lysates also block transportation of NP into the nucleus. K562 lysates $\left(5 \times 10^{5}\right.$ equivalents) were pretreated with $0.5 \mu \mathrm{M}$ GzmK at $37^{\circ} \mathrm{C}$ for $2 \mathrm{~h}$ and then pre-permeabilized HeLa cells with recombinant Myc-NP were added. ERG-absent samples served as negative controls. (d) Double knockdown of $\operatorname{Imp} \alpha 1$ and $\operatorname{Imp} \beta$ decreases the polymerase activity of the influenza virus. $293 \mathrm{~T}$ cells were co-transfected with $\operatorname{sh} \operatorname{lmp} \alpha 1$ and shlmp $\beta$ plasmids $(\operatorname{sh}(\operatorname{Imp} \alpha 1+\operatorname{Imp} \beta))$ for $48 \mathrm{~h}$ and a stable silenced clone was established. Double-silenced 293 T cells were cotransfected with Flu A Pol + NP, pPoll-NS-luc, and pRL-SV40, followed by luciferase assay. (e) tImp $\alpha 1$ and $\operatorname{tmp} \beta$ are not able to rescue polymerase activity. Double-silenced 293 T cells were cotransfected with either full-length importin $\alpha 1$ and importin $\beta$ or their GzmK-truncated fragments, as well as $(\mathrm{Pol}+\mathrm{NP})$ and the luciferase plasmids. ${ }^{* \star} P<0.0001$

\section{Discussion}

Increasing evidence shows that $\mathrm{CD}^{+}{ }^{+} \mathrm{CTLs}$ have pivotal roles in the control of influenza virus infection through TCRmediated cytotoxicity. ${ }^{24,25}$ However, it takes around a week to prime activation of naive $\mathrm{CD}^{+}{ }^{+} \mathrm{T}$ cells for antigen-driven differentiation and proliferation. These findings imply that NK cells may exert a crucial role in the acute control of virus infection during the early stages. NK cells are considered part of the innate immune system, early attackers of both virusinfected cells and transformed tumor cells. ${ }^{26,27}$ Direct killing and cytokine secretion are the two major effector responses of NK cells. Stimulation of NK cells by viruses can produce cytokines such as IFN- $\gamma$, GM-CSF, and TNF- $\alpha$, which cause inflammation, which allows the body to better attack pathogens. NK cells also recognize virus-infected host cells and trigger cell death, resulting in the clearance of infected cells. Interestingly, we found that LAK cells were able to inhibit viral replication and to eliminate the influenza virus.
Although GzmA and GzmB are generally considered to be the most abundant Gzms in humans and mice, ${ }^{5,28}$ cytotoxic granules also contain other Gzms that also exert critical functions in the elimination of viruses and tumors. ${ }^{29} \mathrm{~A}$ previous report showed that the level of circulating GzmK was remarkably elevated in virus-infected patients, implying that GzmK has an important role in viral clearance. ${ }^{30}$ Jenkins et al. ${ }^{8}$ reported that CTLs of gzmA/B ${ }^{-1-}$ mice, which express only GzmK and very limited amounts of GzmC, exhibited effective activity against the influenza virus. Here, we found that in vivo inhibition of GzmK by its specific inhibitor aggravated influenza virus infection. Therefore, Gzms may possess their own specificity with regard to fighting different pathogens.

Like GzmA, GzmK is also a tryptase that induces caspaseindependent cell death. ${ }^{10}$ We have previously demonstrated that GzmK targets the GzmA-associated SET complex and cleaves the known substrates of GzmA in that complex, including SET, Ape1, and HMGB2. ${ }^{10,11}$ However, in addition to sharing some substrates with GzmA, GzmK possesses 
its own substrate specificity. GzmK causes mitochondrial damage by cleavage of Bid, which is not degraded by GzmA. ${ }^{12,31}$ GzmK can cleave tumor suppressor p53 at Lys24 and Lys305 to generate three pro-apoptotic fragments, sensitizing tumor cells to GzmK-mediated cytolysis. ${ }^{14} \mathrm{We}$ determined that VCP is in the SET complex, which is specifically proteolyzed by GzmK to abolish the ERAD pathway, leading to ER stress. ${ }^{13}$ In this study, we eluted importin $\alpha 1$ with the known GzmK substrates SET and VCP (VCP is also in the GzmK-associated SET complex) and found that they were cleaved by GzmK, not other Gzms (data not shown). Importin $\alpha 1$ is one member of the importin $\alpha$ family. This family of proteins all have a structurally conserved $\mathrm{N}$-terminal IBB domain that interacts with a transport partner, importin $\beta$, to generate a ternary import complex for cytoplasmic cargos bearing a classical NLS. ${ }^{21,32}$ It has been reported that NP and PB2 bind to importin $\alpha 1$ through their NLSs for transport into the host cell nucleus through the importin $\alpha 1 / \beta$ dimer. ${ }^{2,20}$ GzmK cleaved importin $\alpha 1$ after Arg $^{13}$ in the N-terminal IBB domain, which is crucial to association with importin $\beta$. Interestingly, the IBB domains of all human importin $\alpha$ isoforms (importin $\alpha 1,3,4,5,6,7$, and 8) are structurally conserved. ${ }^{33}$ The $\mathrm{N}$-terminal Arg residue ( $\mathrm{P} 1$ site) of each isoform is also conserved and the P4-P3-P2-P1 residues are predictively optimal to be cleaved by $\mathrm{GzmK}^{34}$ (Supplementary Figure 1a). Notably, we observed that GzmK cleaved all the other importin $\alpha$ isoforms (WT) in addition to importin $\alpha 1$, whereas GzmK did not proteolyze their P1 sitemutated ones (Supplementary Figure 1b). Moreover, GzmK also degraded importin $\beta$ at its interaction regions in the $\mathrm{C}$ terminus. Thus, GzmK was found to degrade the importin $\alpha 1 / \beta$ dimer. These results indicate that GzmK can inhibit the classical importin $\alpha / \beta$-mediated nuclear import pathway, which ensures blockade of viral protein import to the host nucleus leading to efficient viral clearance.

\section{Materials and Methods}

Cell lines, mice, and viruses. K562 (human erythromyeloblastoid leukemia cell line) cells were maintained in RPMl 1640 medium supplemented with 10\% FBS. A549 (human lung adenocarcinoma cell line), MDCK (dog kidney cell line), HeLa (human epithelial carcinoma cell line), and HEK 293 T (human kidney cell line) cells were grown in DMEM supplemented with $10 \% \mathrm{FBS}, 50 \mu \mathrm{M} \beta$-ME, $100 \mathrm{Units} / \mathrm{ml}$ penicillin, and $100 \mu \mathrm{g} / \mathrm{ml}$ streptomycin. Balb/c mice 4-6-weeks old were purchased from Vital River Laboratories (VRL, Beijing, China). Recombinant influenza virus Flu A/WSN/33 (H1N1) was rescued from 293 T cells through a reverse genetics system and amplified in MDCK cells. Viral infectivity was titrated using a plaque assay.

Antibodies and reagents. Polyclonal antibodies against GzmK were generated by our laboratory. Commercial antibodies included mouse monoclonal antibody for importin $\alpha 1$ (BD Pharmingen, San Jose, CA, USA), importin $\beta$ (BD Pharmingen), FLAG (Sigma-Aldrich, St. Louis, MO, USA), c-Myc (Santa Cruz Biotechnology, Santa Cruz, CA, USA), $\beta$-actin (Sigma-Aldrich), rabbit polyclonal antibodies for SET (Santa Cruz Biotechnology), c-Myc (GenScript USA Inc., Piscataway, NJ, USA), HRP-conjugated sheep anti-mouse $\operatorname{lgG}$ and HRP-conjugated sheep anti-rabbit IgG (Santa Cruz Biotechnology), Alexa 594-conjugated donkey anti-mouse lgG (Molecular Probes, Carlsbad, CA, USA), and Alexa 488-conjugated donkey anti-rabbit lgG (Molecular Probes). Affi-Gel 10 was purchased from Bio-Rad (Hercules, CA, USA). Lipofectamine 2000 was purchased from Invitrogen (Carlsbad, CA, USA). A dual-luciferase reporter assay system was purchased from Promega (Madison, WI, USA).

Mouse infection. For GzmK inhibition, $100 \mu \mathrm{g}$ GzmK-specific inhibitor (KI) was intravenously injected into mice the day before infection. PBS was injected into control group mice. Mice were intranasally infected with Flu A/WSN/33 (H1N1) $\left(10^{2} \mathrm{PFU} /\right.$ mouse) under parenteral anesthesia. All animals were housed in the institute's P2 mouse facility. Animal care and experiments were performed in accordance with the ethics committee guidelines of the Institute of Biophysics (Chinese Academy of Sciences (CAS), Beijing, China).

IHC and in situ hybridization (ISH) assay. The dissected mouse lungs were fixed with $4 \%$ paraformaldehyde, and sectioned for immunohistochemistry $(\mathrm{IHC})$ assay. For in situ hybridization assay, tissue sections were digested with proteinase $\mathrm{K}$ for $5 \mathrm{~min}$, and fixed with $4 \%$ paraformaldehyde for $3 \mathrm{~min}$. Sections were then treated with $0.25 \%$ acetic anhydride for $10 \mathrm{~min}$ and pre-hybridized with salmon sperm DNA at $60^{\circ} \mathrm{C}$ overnight, and then hybridized with the denatured digoxigenin-labeled M2 gene cDNA probe (FluA/WSN/33(H1N1)) for $18 \mathrm{~h}$. The sequence of the $M$ CDNA probe was AAGCTTAAGAGGGAGATAACATTCCA TGGGGCCAAAGAAATAGCACTCAGTTATTCTGCTGGTGCACTTGCCAGTTGTA TGGGCCTCATA.

Plasmid construction. Full-length and GzmK-truncated fragments of human importin $\alpha 1$ were amplified from pcDNA3-FLAG-Imp $\alpha 1$ (kindly gifted by Domenico Delia, Istituto Nazionale Tumori, Italy) and subcloned into pcDNA4/TO/Myc-HisB and $\mathrm{pET} 28 \mathrm{a}$ for eukaryotic and prokaryotic expression. Full-length and GzmKtruncated human importin $\beta$ was amplified from human cDNAs and subcloned into pFLAG-CMV2 and pGEX-4T-1. GzmK cleavage-site mutation plasmids were generated using the Stratagene QuickChange site-directed mutagenesis kit (Stratagene, La Jolla, CA, USA). pET30a-NP was kindly gifted by Dr. Xuemei $\mathrm{Li}$ (Institute of Biophysics), and a c-Myc sequence was inserted into the $\mathrm{N}$ terminus of the NP protein for immunostaining. Ran and NTF/p10 were amplified from human cDNAs and subcloned into pET30a. pCPB1, pCPB2, pCPA, pCNP, pPollNSluc, and pRLSV40 plasmids were used for luciferase assay as described previously. ${ }^{16}$

Expression of recombinant proteins. Recombinant GzmK, mutant S-AGzmK (enzymatically inactive by mutating the active site $\mathrm{Ser}^{214}$ to $\mathrm{Ala}^{214}$ ) were produced and purified as described previously. ${ }^{10}$ Full-length human importin $\alpha 1$, tImp $\alpha 1$, R13A-Imp $\alpha 1$, full-length human importin $\beta, \operatorname{timp} \beta$, Myc-NP, Ran, and NTF/p10 were expressed in Escherichia coli strain BL21 and purified through the Ni-NTA resin for $6 \times$ His-tagged proteins or through the GST resin for GST-tagged proteins.

Affinity chromatography. S-AGzmK was covalently coupled to $1 \mathrm{ml}$ Affi-Gel 10 (Bio-Rad) resin according to the manufacturer's instructions. $1 \times 10^{9} \mathrm{~K} 562$ cells were lysed with lysis buffer (20 mM Tris, pH 7.6, $20 \mathrm{mM} \mathrm{NaCl}, 0.5 \%$ NP-40, $0.5 \mathrm{mM}$ EDTA, $0.5 \mathrm{mM}$ EGTA, $25 \mathrm{mM} \mathrm{KCl}, 5 \mathrm{mM} \mathrm{MgCl} 2,1 \mathrm{mM}$ PMSF, $1 \%$ protease inhibitor cocktail). The lysates were loaded and run through the S-AGzmK affinity column and then eluted through $\mathrm{NaCl}$ concentration gradients in $50 \mathrm{mM}$ Tris- $\mathrm{HCl}, \mathrm{pH} 7.5$. The eluted fractions were resolved by SDS-PAGE and visualized by silver staining.

GzmK loading assay. K562 and HeLa cells were collected and washed three times with HBSS. Cell pellets were resuspended in loading buffer (HBSS with $0.5 \mathrm{mg} / \mathrm{ml} \mathrm{BSA}, 1 \mathrm{mM} \mathrm{CaCl}, 1 \mathrm{mM} \mathrm{MgCl} 2)$. Target cells $\left(2 \times 10^{5}\right)$ in $50 \mu$ l loading buffer were incubated at $37^{\circ} \mathrm{C}$ for the indicated lengths of time with different concentrations of GzmK plus an optimal dose of Ad as described previously. ${ }^{10}$ Cells were incubated for an additional $15 \mathrm{~min}$ in $1 \mathrm{mM}$ PMSF before being lysed for immunoblotting.

GzmK cleavage assay. Recombinant proteins and cell lysates $\left(2 \times 10^{5}\right.$ cell equivalents) in $20 \mu$ l cleavage buffer ( $50 \mathrm{mM}$ Tris, pH 7.6, $1 \mathrm{mM} \mathrm{CaCl}, 1 \mathrm{mM} \mathrm{MgCl}$ ) were incubated at $37^{\circ} \mathrm{C}$ for the indicated lengths of time with different concentrations of GzmK. The reactions were stopped by addition of $5 \times$ SDS loading buffer, and the mixtures were then boiled for $10 \mathrm{~min}$. The samples were resolved by SDS-PAGE and detected by Coomassie brilliant blue staining or immunoblotting with the indicated antibodies.

Co-immunoprecipitation assay. Recombinant proteins or cytosolic lysates $\left(5 \times 10^{6}\right.$ cell equivalents in $150 \mu$ l lysis buffer, $20 \mathrm{mM}$ Tris, $\mathrm{pH} 7.6$, $50 \mathrm{mM} \mathrm{NaCl}, 1 \%$ Triton X-100, 0.1\% EDTA, 0.1\% EGTA) were incubated with the indicated antibodies for $2-6 \mathrm{~h}$ at $4{ }^{\circ} \mathrm{C}$, and the immune complexes were further incubated with protein $A / G$ beads (Santa Cruz Biotechnology) for $2 \mathrm{~h}$ at $4^{\circ} \mathrm{C}$. The immunoprecipitates were washed three times with the wash buffer $(20 \mathrm{mM}$ Tris, $\mathrm{pH} 7.6,150 \mathrm{mM} \mathrm{NaCl}, 0.1 \%$ Triton X-100), mixed with $2 \times$ SDS loading buffer, and boiled for $10 \mathrm{~min}$ before electrophoresis. For other methods, see Supplementary Methods. 
In vitro transportation assay. HeLa cells were grown overnight to subconfluency at $37^{\circ} \mathrm{C}$ in 8-well chamber slides (BD Falcon, BD Biosciences, San Jose, CA, USA). Cells were then washed with pre-cold transport buffer (20 mM HEPES, pH7.3, $110 \mathrm{mM}$ potassium acetate, $5 \mathrm{mM}$ sodium acetate, $1.5 \mathrm{mM}$ magnesium acetate, $1 \mathrm{mM}$ EGTA, $1 \mathrm{mM}$ DTT) and permeabilized on ice with the transport buffer containing $40 \mu \mathrm{g} / \mathrm{ml}$ digitonin for $5 \mathrm{~min}$, and then rinsed twice with the transport buffer to minimize residual proteins in cytoplasm. Cells were then incubated at $30^{\circ} \mathrm{C}$ for $30 \mathrm{~min}$ with $2 \% \mathrm{BSA}, 1 \mu \mathrm{M}$ recombinant Myc-NP protein, transport factors (cytosol extractions or recombinant proteins composed of importin $\alpha 1$, importin $\beta$, Ran-GDP, and NTF/p10), and an energy regeneration system (1 mM ATP, $5 \mathrm{mM}$ creatine phosphate, $20 \mathrm{Units} / \mathrm{ml}$ creatine phosphokinase) in a total volume of $100 \mu \mathrm{l}$. Transported Myc-NP protein in the nucleus was probed through immunostaining with anti-Myc antibody.

Laser-scanning confocal microscopy. Cells were grown on glass slides or centrifuged onto poly-lysine-pretreated glass slides before staining. Cell samples were fixed with $4 \%$ paraformaldehyde at room temperature (RT) for $30 \mathrm{~min}$, and washed 3 times with PBS. Fixed cells were permeabilized with $0.1 \%$ Triton X-100 on ice for 5-10 min and then again washed 3 times with PBS. Primary antibodies against relative proteins were added to the glass slide, incubated at $R T$ for $1 \mathrm{~h}$, and then rinsed with PBS. Samples were then incubated with a secondary Alexa 488-conjugated donkey anti-rabbit IgG or Alexa 594-conjugated donkey anti-mouse IgG antibody for another hour and soaked in $0.1 \mu \mathrm{g} / \mathrm{ml}$ Hoechst for an additional $5 \mathrm{~min}$, and then washed 3 times with PBS. The slides were finally mounted and observed using laser scanning confocal microscopy (Olympus FV500, Tokyo, Japan).

Luciferase assay. WT or importin $\alpha 1$ and importin $\beta$ double-silenced 293 $\mathrm{T}$ cells were plated on 24 -well plates for $18 \mathrm{~h}$ before transfection. pPollNSluc, $\mathrm{PRLSV40}$, pCPB1, pCPB2, pCPA, and pCNP plasmids were transfected together with full-length or GzmK-truncated fragments of importin $\alpha 1$ and importin $\beta$. Cells were harvested and washed twice with PBS at the indicated times. Luciferase assay was performed according to the Promega Dual-Luciferase Reporter Assay system instructions.

\section{Conflict of Interest}

The authors declare no conflict of interest.

Acknowledgements. We thank Dr. Domenico Delia for providing us with importin $\alpha 1$ plasmid and Dr. Xuemei Li for the NP plasmid. We also thank Peng Xue, Zhensheng Xie, Yan Teng, and Chunchun Liu for their technical support. This work was supported by the National Natural Science Foundation of China (30830030, 30972676), 973 Programs (2010CB911902), and the Innovative Program of CAS (XDA01010407, KSCX2-YW-R-42).

\section{Author contributions}

$\mathrm{CZ}$ designed, performed research, analyzed data, and wrote the paper; $\mathrm{CL}$ and $\mathrm{XW}$ performed some research experiments; TT and GG analyzed data; and ZF initiated the study, designed, organized, and wrote the paper.

1. Mattaj IW, Englmeier L. Nucleocytoplasmic transport: the soluble phase. Annu Rev Biochem 1998; 67: 265-306.

2. Gabriel $G$, Herwig A, Klenk HD. Interaction of polymerase subunit PB2 and NP with importin alpha1 is a determinant of host range of influenza A virus. PLoS Pathog 2008; 4: e11.

3. Fan Z, Zhang Q. Molecular mechanisms of lymphocyte-mediated cytotoxicity. Cell Mol Immunol 2005; 2: 259-264.

4. Chowdhury D, Lieberman J. Death by a thousand cuts: granzyme pathways of programmed cell death. Annu Rev Immunol 2008; 26: 389-420.

5. Fan Z, Beresford PJ, Oh DY, Zhang D, Lieberman J. Tumor suppressor NM23-H1 is a granzyme A-activated DNase during CTL-mediated apoptosis, and the nucleosome assembly protein SET is its inhibitor. Cell 2003; 112: 659-672.

6. Topham DJ, Tripp RA, Doherty PC. CD8+ T cells clear influenza virus by perforin or Fasdependent processes. J Immunol 1997; 159: 5197-5200.

7. Regner M, Pavlinovic L, Koskinen A, Young N, Trapani JA, Mullbacher A. Cutting edge: rapid and efficient in vivo cytotoxicity by cytotoxic $\mathrm{T}$ cells is independent of granzymes $\mathrm{A}$ and B. J Immunol 2009; 183: 37-40.
8. Jenkins MR, Trapani JA, Doherty PC, Turner SJ. Granzyme K expressing cytotoxic T lymphocytes protects against influenza virus in granzyme AB-/- mice. Viral Immunol 2008; 21: 341-346.

9. Bratke K, Kuepper M, Bade B, Virchow Jr JC, Luttmann W. Differential expression of human granzymes $A, B$, and $K$ in natural killer cells and during $C D 8+T$ cell differentiation in peripheral blood. Eur J Immunol 2005; 35: 2608-2616.

10. Zhao T, Zhang H, Guo $\mathrm{Y}$, Zhang Q, Hua G, Lu H et al. Granzyme $\mathrm{K}$ cleaves the nucleosome assembly protein SET to induce single-stranded DNA nicks of target cells. Cell Death Differ 2007; 14: 489-499.

11. Guo $Y$, Chen J, Zhao T, Fan Z. Granzyme $K$ degrades the redox/DNA repair enzyme Ape1 to trigger oxidative stress of target cells leading to cytotoxicity. Mol Immunol 2008; 45 : 2225-2235.

12. Zhao T, Zhang H, Guo Y, Fan Z. Granzyme K directly processes bid to release cytochrome $\mathrm{c}$ and endonuclease $\mathrm{G}$ leading to mitochondria-dependent cell death. J Biol Chem 2007; 282: 12104-12111.

13. Guo Y, Chen J, Shi L, Fan Z. Valosin-containing protein cleavage by granzyme $K$ accelerates an endoplasmic reticulum stress leading to caspase-independent cytotoxicity of target tumor cells. J Immunol 2010; 185: 5348-5359.

14. Hua G, Wang S, Zhong C, Xue P, Fan Z. Ignition of $p 53$ bomb sensitizes tumor cells to granzyme K-mediated cytolysis. J Immunol 2009; 182: 2152-2159.

15. Harari A, Enders FB, Cellerai C, Bart PA, Pantaleo G. Distinct profiles of cytotoxic granules in memory CD8 T cells correlate with function, differentiation stage, and antigen exposure. $J$ Virol 2009; 83: 2862-2871.

16. Toyoda $T$, Hara K, Imamura Y. Ser624 of the PA subunit of influenza A virus is not essential for viral growth in cells and mice, but required for the maximal viral growth. Arch Virol 2003; 148: 1687-1696.

17. Bovenschen N, Quadir R, van den Berg AL, Brenkman AB, Vandenberghe I, Devreese B et al. Granzyme $\mathrm{K}$ displays highly restricted substrate specificity that only partially overlaps with granzyme A. J Biol Chem 2009; 284: 3504-3512.

18. Zhang J, Rubio V, Lieberman MW, Shi ZZ. OLA1, an Obg-like ATPase, suppresses antioxidant response via nontranscriptional mechanisms. Proc Natl Acad Sci USA 2009; 106: 15356-15361.

19. Goldfarb DS, Corbett AH, Mason DA, Harreman MT, Adam SA. Importin alpha: a multipurpose nuclear-transport receptor. Trends Cell Biol 2004; 14: 505-514.

20. Wang P, Palese P, O'Neill RE. The NPI-1/NPI-3 (karyopherin alpha) binding site on the influenza a virus nucleoprotein NP is a nonconventional nuclear localization signal. $J$ Virol 1997; 71: $1850-1856$

21. Lott $\mathrm{K}$, Cingolani $\mathrm{G}$. The importin beta binding domain as a master regulator of nucleocytoplasmic transport. Biochim Biophys Acta 2010; 1813: 1578-1592.

22. Cingolani G, Petosa C, Weis K, Muller CW. Structure of importin-beta bound to the IBB domain of importin-alpha. Nature 1999; 399: 221-229.

23. Cros JF, Palese P. Trafficking of viral genomic RNA into and out of the nucleus: influenza, Thogoto and Borna disease viruses. Virus Res 2003; 95: 3-12.

24. Doherty PC, Topham DJ, Tripp RA, Cardin RD, Brooks JW, Stevenson PG. Effector CD4+ and CD8+ T-cell mechanisms in the control of respiratory virus infections. Immunol Rev 1997; 159: 105-117.

25. Jenkins MR, Kedzierska K, Doherty PC, Turner SJ. Heterogeneity of effector phenotype for acute phase and memory influenza A virus-specific CTL. J Immunol 2007; 179: 64-70.

26. Vivier E, Raulet DH, Moretta A, Caligiuri MA, Zitvogel L, Lanier LL et al. Innate or adaptive immunity? The example of natural killer cells. Science 2011; 331: 44-49.

27. Fan Z, Yu P, Wang Y, Fu ML, Liu W, Sun $Y$ et al. NK-cell activation by LIGHT triggers tumor-specific CD8+ T-cell immunity to reject established tumors. Blood 2006; 107: 1342-1351.

28. Voskoboinik I, Smyth MJ, Trapani JA. Perforin-mediated target-cell death and immune homeostasis. Nat Rev Immunol 2006; 6: 940-952.

29. Bovenschen N, Kummer JA. Orphan granzymes find a home. Immunol Rev 2010; 235 117-127.

30. Bade B, Lohrmann J, ten Brinke A, Wolbink AM, Wolbink GJ, ten Berge IJ et al. Detection of soluble human granzyme $\mathrm{K}$ in vitro and in vivo. Eur J Immunol 2005; 35 2940-2948.

31. Martinvalet D, Zhu P, Lieberman J. Granzyme A induces caspase-independent mitochondrial damage, a required first step for apoptosis. Immunity 2005; 22: $355-370$.

32. Lange A, Mills RE, Lange CJ, Stewart M, Devine SE, Corbett AH. Classical nuclear localization signals: definition, function, and interaction with importin alpha. J Biol Chem 2007; 282: 5101-5105.

33. Kelley JB, Talley AM, Spencer A, Gioeli D, Paschal BM. Karyopherin alpha7 (KPNA7), a divergent member of the importin alpha family of nuclear import receptors. BMC Cell Biol 2010; 11: 63-74.

34. Mahrus S, Craik CS. Selective chemical functional probes of granzymes A and B reveal granzyme $B$ is a major effector of natural killer cell-mediated lysis of target cells. Chem Biol 2005; 12: 567-577. 